\title{
LA LENGUA MATERNA DE LA POBLACIÓN COMPOSTELANA
}

\author{
por \\ MAURO FERNÁNDEZ
}

\section{Introducción}

La expresión "lengua materna", a primera vista bastante transparente en su significación, no escapa a las confusiones y ambigüiedades terminológicas que atraviesan todas las ciencias, y muy especialmente las llamadas ciencias ciencias sociales. Aquí la uso en su sentido más inmediato, como lengua inicial de una persona, como lengua en la que uno aprende a hablar. Solamente en este sentido puede considerarse la lengua materna como una invariante ${ }^{1}$.

\footnotetext{
${ }^{1}$ Skutnabb-Kangas $(1984,1988)$ define la lengua materna de cuatro modos diferentes: según el origen (la lengua aprendida primero), según la competencia (la lengua que se domina mejor), según la función (la lengua que más se usa) y según la identificación (interna: la lengua con la que uno se identifica, y externa: la lengua que los demás nos atribuyen como nativa). Naturalmente, esta manera de ver las cosas lleva consigo el que la lengua materna no sea una invariante; la misma persona puede tener diferentes lenguas maternas, dependiendo de cuál de las definiciones se utilice, y puede cambiar de lengua materna varias veces en su vida, excepto en la acepción que toma el origen como criterio definitorio.
}

"CUADERNOS DE ESTUDIOS GALLEGOS", Tomo XXXIX, Fascículo 104, Santiago 1991. 
Es práctica habitual, en la sociología del lenguaje, que las opciones de respuesta a la pregunta sobre la lengua materna (LM) sean solamente dos, o tantas como lenguas en las situaciones de multilingüismo, como si no fuese posible que una persona tenga más de una LM. Aunque este planteamiento sea correcto en muchas situaciones, pienso que, en ciertas condiciones, es posible que haya un importante sector de la población que tenga más de una LM. Ello es relativamente frecuente en el nivel individual del bilingüismo, en los casos de niños cuyos padres tienen LMs diferentes y cada uno utiliza la suya para dirigirse a los hijos -aunque una de ellas sea la habitual en la comunicación de los padres entre sí. Esta situación está ampliamente documentada en la bibliografía sobre el bilingüismo. Ahora bien, cuando la distancia entre dos sistemas lingüísticos en contacto no es muy grande, de tal modo que existe un alto grado de intercomprensión entre sus usuarios, y cuando existe un bilingüismo generalizado en el medio ambiente del niño, especialmente en su ámbito familiar -bien porque las personas que mantienen estrecho contacto con el niño en la época de su aprendizaje lingüístico usan ambas lenguas, bien porque algunas de estas personas usan preferentemente o siempre una lengua, y otras usan siempre o preferentemente la otra- el número de bilingües desde que comienzan a hablar podrá ser bastante elevado, trascendiendo así el nivel individual o el simplemente anecdótico. La mejor confirmación posible de que se puede tener más de una lengua la constituyen las respuestas de los encuestados cuando se incluye en el cuestionario la opción de respuesta adecuada. La mayoría de los cuestionarios utilizados en Galicia no han prestado la debida atención a este aspecto; a veces, por una generalización injustificada de la propia experiencia personal del autor del cuestionario (a quien tiene solamente una lengua materna le resulta difícil imaginar que no a todo el mundo le sucede lo mismo), y a veces por motivos piadosos, pero igualmente injustificados: es evidente, para cualquiera familiarizado con los procesos de sustitución linguística, que los bilingües iniciales (BI) procederán mayoritariamente de la lengua que ocupe la posición más débil en el incesante batallar de las lenguas en contacto. Por consiguiente, eliminar la posibilidad, en el caso de Galicia, de decir que se ha aprendido a hablar en las dos lenguas hace que aumente (artificialmente) el porcentaje de gallego-hablantes iniciales (GHI). Naturalmente, la realidad es mucho más tenaz que los enmasacaramientos, voluntarios o no, que de ella hagamos. Peor aún, los enmascaramientos y los tópicos pueden tener un efecto contrario al que persiguen quienes los hacen y difunden. ¿No es, acaso, 'vox populi' que el gallego es la lengua mayoritaria en Galicia? ¿No es eso lo que frecuentemente difundimos fuera de ella? Y si esto es así, ¿para qué se necesitan medidas especiales que protejan la lengua y eviten su desaparición?

"CUADERNOS DE ESTUDIOS GALLEGOS", Tomo XXXIX, Fascículo 104, Santiago 1991. 
Pero es el caso que ya Murguía, en 1906, en el discurso inaugural de la Real Academia Gallega tuvo que reconocer el enorme retroceso que había sufrido el gallego desde los tiempos de su niñez; y, desde ese discurso hasta hoy, muchas han sido las quejas por el mismo motivo. ¿Cómo es posible, entonces, que el Informe FOESSA (1970) asegure que habla gallego más del $70 \%$ de la población? Ya a comienzos de siglo se manejaba un porcentaje similar, si bien de un modo intuitivo, sin base en datos estadísticos ${ }^{2}$. ¿Cómo es, pues, posible que siga hablando gallego el $70 \%$ de la población si el retroceso ha sido constante? O bien no ha habido el retroceso denunciado en múltiples ocasiones, o bien las afirmaciones que leemos y oímos con frecuencia acerca del porcentaje de gallego-hablantes no responden a la realidad, siendo simplemente creencias ancladas en el pasado. Estas afirmaciones se apoyan a veces en datos estadísticos sacados de su contexto, mal interpretados o, simplemente, mal recogidos por los investigadores. (Pensemos que una pregunta como "¿habla usted gallego?" puede ser respondida afirmativamente tanto por quien se expresa siempre o casi siempre en esa lengua como por quien, teniendo como lengua habitual el castellano, es capaz de usar y usa el gallego esporádicamente). Pero aún cuando los investigadores distingan cuidadosamente entre éste y otros matices, el tópico se alimenta -como siempre sucede- de los datos menos elaborados. Por citar sólo algunos de los casos más frecuentes, además de la no distinción entre posibilidad de uso y uso real, desde el tópico no se distingue entre los diversos tipos de hábitat, o cuando sí se hace, se deduce que por ser abrumadoramente mayoritaria la población rural -¿no será éste otro tópico?- y por haberse mantenido ésta más leal al gallego, la lengua no corre peligro, sin percatarse de que, también en Galicia, las ciudades son el motor de la dinámica de los cambios socioculturales, y de que en ellas el predominio del castellano es más que evidente desde hace tiempo. Y sobre todo, no se suele distinguir entre generaciones; aún admitiendo que el $70 \%$ de la población hable gallego -lo que dista de ser cierto si nos referimos a la lengua habitual- ¿cuál es la situación en las generaciones más jóvenes, de quienes depende, en definitiva, el futuro de la lengua? Más concretamente, atendiendo a la dirección de la dinámica sociocultural, ¿cuál es la situación en las generaciones urbanas más jóvenes?

Intentando responder a estas preguntas, inicié en 1981 una investigación con el objeto fundamental de aportar datos (recogidos y analizados con más cuidado que el habitual) sobre la relación de fuerzas entre el gallego y el castellano en los niños compostelanos, en lo concerniente a la

\footnotetext{
${ }^{2}$ Véase M. Fernández, 1978, p. 112.
} 
lengua materna, uso, conocimiento y actitudes lingüísticas ${ }^{3}$. Los niños de entonces son hoy adultos o, cuando menos, jóvenes adultos que tienen entre 19 y 23 años. Por razones de comodidad, me referiré a ellos sistemáticamente como "jóvenes". Pues bien, basta de preámbulo y veamos ya cuál es la lengua incial de estos jóvenes.

\section{Datos generales}

Los resultados generales obtenidos (figura 1) difieren considerablemente de los de otras encuestas realizadas en Galicia. Aunque los datos no sean directamente comparables, por proceder de muestras que representan sectores de población distintos y de mayor amplitud geográfica, es necesario destacar el bajo porcentaje de gallego-hablantes iniciales (GHI)

\section{LENGUA MATERNA TOTAL DE LA MUESTRA}

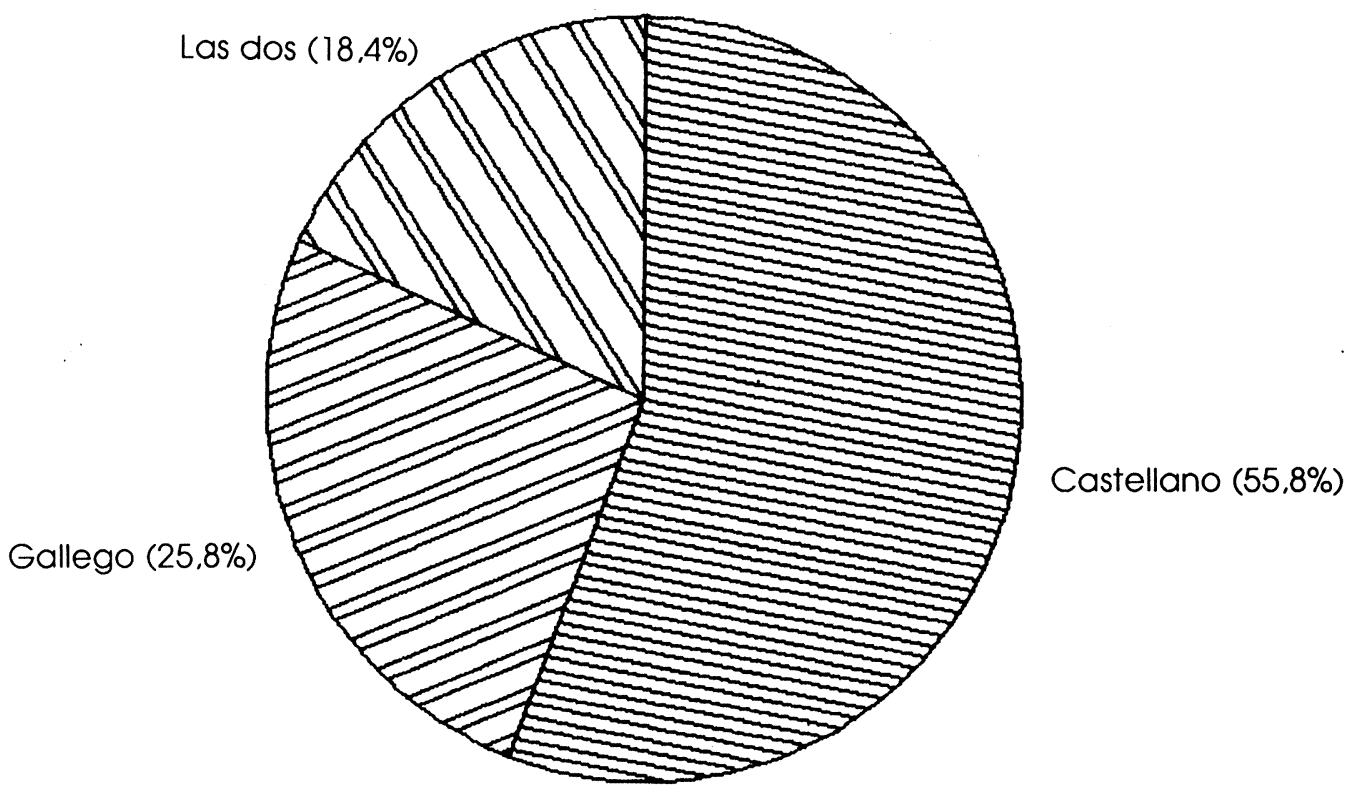

${ }^{3}$ Los resultados de este estudio fueron presentados como Tesis Doctoral (M. Fernández, 1984). La muestra, con un diseño por conglomerados, consistió en 837 alumnos de EGB, con edades entre diez y catorce años, de nueve centros escolares. El tamaño de la muestra es mucho mayor que lo que se acostumbra; supone el 7,9\% del total de la población de EBG en 1981, y el $19 \%$ de los matriculados en los cursos 5, $6^{\circ}$ y $7^{\circ}$. Par una descripción detallada de la muestra, véase Fernández (1984), po. 14-

"CUADERNOS DE ESTUDIOS GALLEGOS", Tomo XXXIX, Fascículo 104, Santiago 1991. 
en la juventud de Compostela: solamente la cuarta parte de los jóvenes tiene el gallego como LM; incluso si consideramos incluidos en esta categoría a los que responden "las dos", nos situamos en un 44,6\%, mientras que en el resto de las encuestas el porcentaje se sitúa casi siempre por encima del 70\% ${ }^{4}$. Del Campo et al. (1977) obtienen un 69\% de GHI para el conjunto de la población gallega ${ }^{5}$. El informe FOESSA (1970) nos da un $76 \%$ de amas de casa galegas que aprendieron a hablar primero en gallego, frente a un $24 \%$ que lo hicieron en castellano. Más favorables al gallego todavía son los resultados de Ayestarán y de la Cueva (1974) para las amas de casa de la provincia de Pontevedra: son GHI el $82 \%$, porcentaje que se eleva al $92 \%$ si excluimos a las amas de casa del municipio de Vigo.

Los datos sobre esta última localidad son especialmente importantes para nosotros, por tratarse de un municipio fundamentalmente urbano. Aunque sea diferente de Santiago en aspectos importantes, no parece haber razones de peso para pensar que hace unos cuantos años, en el momento en que las amas de casa encuestadas por Ayestarán y de la Cueva aprendieron a hablar, estuviese Vigo menos castellanizado que Santiago ${ }^{6}$. Podemos, pues, considerar el porcentaje de GHI del municipio de Vigo

${ }^{4}$ Me refiero a las encuestas relalizadas entre la población adulta. En una encuesta posterior a la mía, encargada por la Dirección Xeral de Política Lingüística (1986), con una muestra de casi dos mil escolares de EGB de toda Galicia, se obtiene un $41,9 \%$ de GHI y un $55,8 \%$ de CHI (2,3\% responden "otras"). Parte de las diferencias entre estos resultados globales y los obtenidos en Santiago podrían deberse al distinto peso de las submuestras rurales respectivas: $39,3 \%$ en la encuesta de la Dirección Xeral y 29,2\% en la mía; de todos modos, la diferencia en la composición de la muestra no es tan grande como para explicar por sí sola la diferencia de resultados. Podríamos pensar que "rural" no tiene el mismo sentido aplicado a los niños de A Sionlla y Labacolla (zonas rurales del municipio de Santiago) que a los de las aldeas cercanas a Laza, Cualedro, Samos, etc. Más adelante me ocuparé de este aspecto. Pero, en mi opinión, la fuente mayor de divergencias bien podría ser el que en el cuestionario de la Dirección Xeral se sigue la tendencia habitual de descartar la posibilidad de un bilingüismo inicial, lo que repercute, como hemos visto, en un aumento del porcentaje de GHI.

${ }^{5}$ Debe de haber un error en este dato, ya que el porcentaje de $\mathrm{CHI}$ en esta encuesta es el 33,7\%.Como sugiere Rojo (1981), que es quien pone de manifiesto la errata, podría tratarse del $66 \%$ y $33,7 \%$, o bien del $69 \%$ y $30,7 \%$.

${ }^{6}$ Solamente el 7,7\% de las amas de casa encuestadas por Ayesterán y de la Cueva tenía menos de veinticinco años en el momento de la encuesta, y el 10,3\% tenía más de sesenta y cinco. Por consiguiente, el $82 \%$ tenía una edad entre los veinticinco y los sesenta y cinco años (y, entre treinta y cinco y setenta y cinco en el momento de mi encuesta). Por otra parte, la edad de la mayoría de los padres de la población de mi encuesta se sitúa entre los reinta y cinco y los cincuenta años y tenían, por consiguiente, entre veinticinco y cuarenta en el momento en que Ayesterán y de la Cueva recogieron sus datos. Podría pensarse que no se debe esti- 
como una estimación aproximada del de Santiago en la generación de los padres ${ }^{7}$. El porcentaje de GHI en el municipio de Vigo es el 64\%, dos veces y media superior al de los jóvenes objeto de este estudio ${ }^{8}$.

\section{Lengua materna y hábitat}

El panorama esbozado en el punto anterior cambia un poco si tomamos en consideración la distribución de la LM según el tipo de hábitat. Como se puede observar en la figura 2, en la ciudad hay un evidente pre-

LENGUA MATERNA SEGÚN EL HÁBITAT

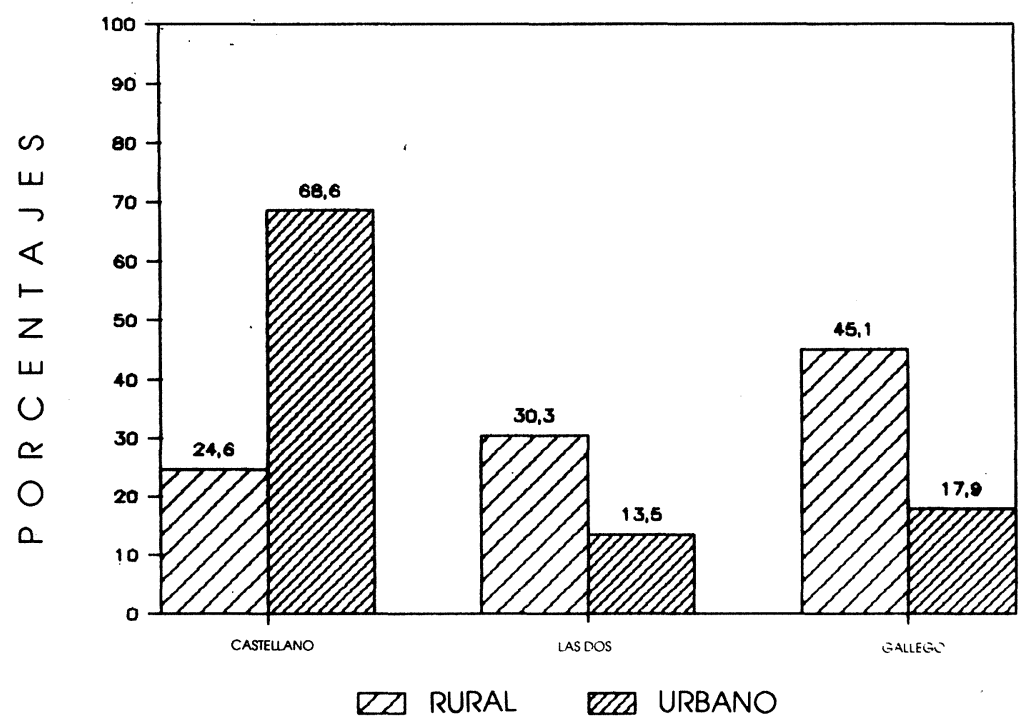

mar el porcentaje de GHI entre los padres de mi encuesta a partir de los datos de Vigo, ya que el rango de edades de Ayesterán y de la Cueva es más amplio; además, desconozco si la submuestra de Vigo presentaba algún sesgo hacia los de mayor o menor edad. Incluso así, téngase en cuenta que el porcentaje de GHI para el conjunto de la provincia de Pontevedra, entre las amas de casa menores de veinticinco años era, en 1974 , el $81 \%$.

${ }^{7}$ Preguntar directamente a los encuestados, especialmente si son niños, cuál es la LM de sus padres no es un buen procedimiento, ya que existe una alta probabilidad de que se desconozca este dato y se dé una respuesta a partir del uso observado, lo que podría llevar a una sobrevaloración del castellano.

${ }^{8}$ Hay que tener en cuenta que la no inclusión en estas encuestas de una opción de respuesta que permita detectar a los BI hace difícil la comparación. Es muy posible que los porcentajes de GHI hubiesen resultado más bajos y, por consiguiente, la desgalleguización aparecería como menor en el paso de una generación a otra, pero no menor en términos absolutos: simplemente, habría comenzado antes. Quien, pese a todo lo dicho en la introducción, prefiera datos que alienten su optimismo, puede sumar los $\mathrm{BI}$ a los $\mathrm{GHI}$; pero aun así la desgalleguización en lo que a lengua materna se refiere resulta importante.

"CUADERNOS DE ESTUDIOS GALLEGOS", Tomo XXXIX, Fascículo 104, Santiago 1991. 
dominio del castellano como LM, con el 68,8\%; menos de la quinta parte (el 17,9\%) tienen como tal el gallego, y un 13,5\% declara haber aprendido a hablar simultáneamente en las dos lenguas. Estos porcentajes contrastan claramente con los obtenidos en las zonas rurales del municipio, en donde el porcentaje más elevado corresponde al gallego, con el $45,1 \%$. frente al $24,6 \%$ de LM castellana; el 30,3\% restante aprendió a hablar en las dos 9 .

Ante la falta de datos correspondientes a la generación de los padres en el municipio compostelano, acudo una vez más a los suministrados por Ayestarán y de la Cueva (1974) para el municipio de Vigo. En las zonas rurales del municipio obtienen estos autores un $77 \%$ de GHI, frente a un 23\% de amas de casa con el castellano como LM. En otras zonas rurales los porcentajes son más elevados: un $90 \%$ en la comarca de la Ría de Arosa perteneciente a la provincia de Pontevedra y un $95 \%$ en el lado coruñés de la misma ría aprendieron a hablar primero en gallego. Estos datos parecen indicar que no se puede asumir, sin más, una homogeneidad lingüística en las zonas rurales gallegas, y que la distancia a la ciudad juega un papel bastante importante en la configuración sociolingüística, al menos en lo que se refiere a la distribución de la lengua inicial. Más adelante veremos, sin embargo, que la distancia no es un factor que por sí sólo explique estas diferencias, sino que actúa solamente en conjunción con otros factores.

Volviendo a los datos de las zonas rurales del municipio de Vigo, y asumiendo una vez más una hipotética homogeneidad del Vigo rural con el Santiago rural en la época en la que los padres de los jóvenes de nuestra muestra aprendieron a hablar ${ }^{10}$, se observa un importante descenso del

${ }^{9} \underline{\mathrm{Ji}}^{2}=136,32$ (signif. $=0,0000$ ). Para ajustar las tablas al número de casos y poder compararlas, utilicé $\underline{V}$ de Cramer, que en este caso es 0,40 . En la encuesta de la Dirección Xeral de Política Lingüística (1986) se obtienen resultados todavía más negativos para el gallego en las ciudades: son $\mathrm{CHI}$ el $97,7 \%$ de los que residen en el centro de las ciudades, el $84,2 \%$ de los que viven en barrios y el $88 \%$ de los que viven en los alrededores (este último concepto no está definido). Parte de estas diferencias pueden deberse a que la correlación entre lugar de nacimiento (utilizado en mi encuesta) y lugar de residencia (utilizado por la DXPL) no es perfecta ni mucho menos. Por ejemplo, sólo el $22 \%$ de los que viven en los alrededores nacieron en ellos, y el $27,1 \%$ de los que viven en la ciudad no nacieron en ella ni en los alrededores. La edad puede ser también un factor importante, pues, aunque las dos recogidas de datos están separadas sólo por tres años, los efectos de la desgalleguización en la LM son tan intensos que se pueden apreciar con intervalos de solamente un año, como mostraré más adelante.

${ }^{10}$ Un procedimiento indirecto, a partir de mis datos, refuerza la suposición de esta homogeneidad. Podemos suponer, razonablemente, que el gallego fue la lengua inicial de, al menos, aquellos padres que hablan entre sí única o fundamentalmente en gallego; el porcentaje correspondiente es el $73,4 \%$, muy próximo al que obtie-

"CUADERNOS DE ESTUDIOS GALLEGOS", Tomo XXXIX, Fascículo 104, Santiago 1991 
porcentaje de GHI: de un $77 \%$ a un $45,1 \%$. Pero es muy importante tener en cuenta que ese descenso no repercute en un aumento del porcentaje de castellano-hablantes iniciales (CHI): $23 \%$ en los datos de Ayestarán y de la Cueva y $24,6 \%$ en nuestros datos. El descenso de GHI es absorbido, casi en su totalidad, por el grupo de BI, que representa el 30,3\% de los jóvenes rurales de mi muestra. Este dato parece confirmar lo acertado de haber incluido esta categoría en la encuesta.

Más importanes son las pérdidas sufridas por el gallego en cuanto LM de los jóvenes urbanos. Ayestarán y de la Cueva obtienen en Vigo ciudad un 55\% de GHI, frente a un $43 \%$ de CHI. La comparación de estos datos con los de los jóvenes compostelanos ${ }^{11}$ muestra un descenso de treinta y siete puntos en el porcentaje de GHI (del 55\% al 17,9\%), descenso que, a diferencia de lo que pudimos observar en los jóvenes rurales, repercute decisivamente en el porcentaje de encuestados cuya LM es el castellano, que del $43 \%$ en los datos de Vigo se eleva a un $68,8 \%$; el porcentaje de BI es, por consiguiente, menor en la ciudad que en el campo: $13,5 \%$ frente al $30,3 \%$.

Según Lieberson (1980), el mecanismo principal de desaparición de una lengua a costa de la expansión de otra es el cambio de la LM de la población. De ser esto así, los datos presentados indicarían una fuerte aceleración del proceso de desgallegización: el descenso de GHI en el plazo de una generación supera los treinta puntos, alcanzando los treinta y siete en el caso de la ciudad. No obstante, como he argumentado en un trabajo anterior (Fernández, 1983), en Galicia no es la LM el mejor dato para cuantificar la desgallegización, sino el uso y, a partir de éste, la desgallegización es considerablemente menor de lo que deduciría de los datos presentados hasta ahora.

nen Ayestarán y de La Cueva en las zonas rurales del municipio de Vigo. Digo al menos, porque un $22,5 \%$ de los padres usa ambas lenguas por igual $\mathrm{y}$, con toda probabilidad, hay entre estos últimos GHI, en una proporción difícil de determinar.

11 Aplicando el procedimiento indirecto expuesto en la nota anterior, el gallego fue la lengua inicial de, al menos el $34,4 \%$ de los padres de los jóvenes urbanos encuestados, ya que éste es el porcentaje de quienes la usan como lengua única o fundamental de comunicación en la pareja. Como he mostrado en otro lugar (Fernández, 1983), el ritmo de la desgalleguización en el uso fue muy importante en los padres urbanos; por ello, no es demasiado aventurado considerar como GHI al $20,1 \%$ de padres que usan ambas lenguas por igual. El posible error en esta apreciación queda, en mi opinión, compensado por el hecho de que, sin duda, algunos de los padres urbanos que usan única o fundamentalmente castellano son gallegohablantes de origen. Se puede, pues, situar el porcentaje de GHI en Santiago ciudad 
Por otra parte, ya hemos visto cómo en el campo este descenso no redunda en un aumento del porcentaje de CHI, sino en el de BI. Indudablemente, este grupo implica la existencia de grados en la desgallegización.

Cabría pensar que una castellanización parcial es, en el fondo, una fuerza de resistencia a favor del gallego; si la estrategia lingüística de los padres que enseñan ambas lenguas a sus hijos estuviese orientada pura y simplemente hacia el abandono lingüístico, cabe preguntarse por el porqué de mantener las dos lenguas. Tal vez nos encontremos ante una actitud ambivalente en los padres, quienes, por una parte, tratan de evitar un posible fracaso escolar de sus hijos en un sistema educativo en el que prevalece el castellano, y de prepararlos para el éxito en una sociedad en la que, hasta el momento, no ha sido posible lograrlo sin el conocimiento de esta lengua; por otra parte, se resisten a que sus hijos abandonen el gallego, porque ello supondría excluirlos de las redes sociales en las que están inmersos. En Fernández (1984) he mostrado cómo la mayoría de estos jóvenes usan ambas lenguas por igual en la comunicación con sus padres y cómo, en el hábitat rural, son bastantes más los BI que usan única o fundamentalmente gallego que los que usan única o fundamentalmente castellano.

Es posible, sin embargo, que en algunos casos el bilingüismo inicial no sea más que un fracaso en el intento de enseñar a los jóvenes el castellano; es decir, la estrategia sería la del abandono abandono lingüístico, que no se consuma, bien por el deficiente dominio del castellano en los padres, bien por el esfuerzo que supone mantener las dos lenguas completamente separadas en el hogar cuando se está instalado en una de ellas. A este respecto es significativo que el mayor porcentaje de BI se dé en el hábitat rural, como ya he dicho. Viendo ahora los datos desde otra prespectiva, resulta que el $48,1 \%$ de los jóvenes BI son rurales, a pesar de que éstos representan solamente el 29,2\% de la muestra; el 51,9\% de los jóvenes que aprendieron a hablar en las dos lenguas son urbanos, cuando esperaríamos que lo fuera un $70,8 \%$, de no haber diferencias de distribución asociadas con el hábitat. En el punto siguiente veremos, además, cómo el porcentaje de BI en la clase social baja es superior al observado en las otras clases sociales.

Resulta difícil, sólo con estos datos, optar por una de estas interpretaciones. Lo más probable es que en esta categoría confluyan los resultados de las dos posturas -y, tal vez, de alguna más. De todos modos, lo expuesto muestra su complejidad e importancia, así como la necesidad de prestarle una atención especial en los estudios que en adelante se lleven a cabo.

Páginas atrás me refería a la heterogeneidad lingüística del medio rural gallego y sugería la posible influencia de la distancia a la ciudad en los 
porcentajes de GHI. Ello explicaría la diferencia entre los diversos porcentajes suministrados por Ayestarán y de la Cueva, que van desde el $77 \%$ en las zonas inmediatas a Vigo hasta el $95 \%$ en la ribera coruñesa de la Ría de Arosa. Sin embargo, según datos de Pérez de Guzmán (1974), en esa misma Ría el porcentaje de GHI entre los hijos de las familias mariscadoras es sólo el $63 \%$, como máximo ${ }^{12}$.

No es la diferencia entre estos datos y los de Ayestarán y de la Cueva lo que llama la atención, diferencia, por otra parte, perfectamente explicable por tratarse de generaciones diferentes, sino la diferencia de estos datos con los de Santiago; en una zona inmediata a la ciudad encontramos un porcentaje de $\mathrm{CHI}$ inferior al de una zona sensiblemente más alejada: el 24,6\% en las zonas rurales del municipio de Santiago frente al $36 \%$ entre las familias mariscadoras de la Ría de Arosa. Parece, pues, que la distancia a la ciudad no es el factor decisivo. Es más: ni siquiera una misma zona rural es necesariamente homogénea en su situación sociolingüística, según se desprende de los datos que presento a continuación.

En la figura 3 aparecen los porcentajes correspondientes a la LM de los jóvenes rurales de nuestra muestra según la profesión de los padres. Las tres actividades profesionales presentadas en el gráfico incluyen a los padres del $89,3 \%$ de los jóvenes rurales; el $11,7 \%$ restante se distribuye entre varias categorías profesionales (funcionarios, comerciantes, profesiones liberales, etc.), con escasas frecuencias en cada una de ellas, por lo que me pareció oportuno exluirlos de este análisis.

Tal vez sorprenda el bajo porcentaje de padres dedicados a los trabajos agrícolas: el 25,4\%, en contraste con la imagen de una Galicia rural fundamentalmente campesina. No obstante, hay que tener en cuenta la proximidad de una ciudad que, si bien no se puede catalogar como zona industrial, cuenta con algunas fábricas en los alrededores que absorben mano de obra procedente de las aldeas cercanas. Por otra parte, es muy proba-

12 Esta afirmación debe tomarse con suma cautela. No he podido consultar el trabajo de Pérez Guzmán. Pero Ayesterán y de la Cueva lo comentan y reproducen el siguiente párrafo: "A tenor del cuadro (se refiere a un cuadro sobre uso. M.F.) el castellano ve reducido su uso hablado entre los adultos a un 5\% aproximadamente, mientras que se emplea para hablar a la generación nueva en un tercio largo de los casos. He ahí la señal de que el idioma gallego, hasta ahora irreductible, está perdiendo terreno con vistas a los años por venir". (Cit. en la p. 105 de Ayesterán y de la Cueva). Aunque Pérez Guzmán no se refiere a la LM, si un tercio de la familias responde que usa el castellano para dirigirse a sus hijos (exactamente, el 36\%), y si tenemos en cuenta que las opciones de respuesta a su pregunta sobre uso fueron solamente dos, "gallego" y "castellano", podemos deducir que en la mayoría de estos casos lo han usado desde el nacimiento de los hijos. Lo que pueda haber de erróneo en esta deducción quedará contrarrestado, creo, por el proceso de signo in-

"CUADERNOS DE ESTUDIOS GALLEGOS", Tomo XXXIX, Fascículo 104, Santiago 1991. 


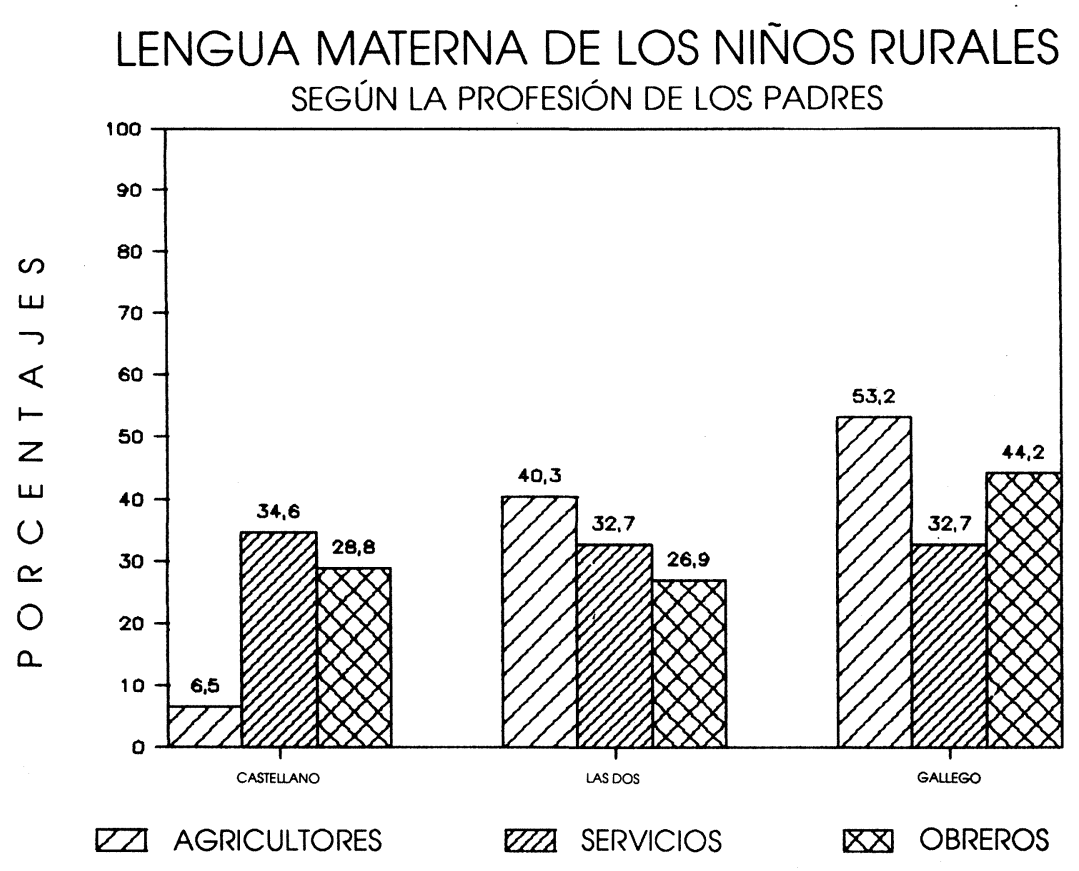

ble que una importante proporción de los trabajadores incluidos entre los obreros (42,6\% de la muestra) sean agricultores a tiempo parcial, en horas libres, fines de semana, períodos vacacionales o de paro. Las profesiones que figuran en el gráfico se refieren, pues, a la actividad principal.

El porcentaje de $\mathrm{CHI}$ entre los hijos de agricultores es solamente el $6,5 \%$, muy por debajo del promedio de la zona rural estudiada. A considerable distancia, y ya por encima del promedio, se encuentran los hijos de obreros, con un $28,8 \%$ y, por último, los hijos de trabajadores del sector servicios, con un 34,6\%. Correlativamente, el porcentaje de GHI supera el promedio rural entre los hijos de agricultores, con un 53,2\%, seguido de los hijos de obreros, con un $44,2 \%$ y, bastante por debajo del promedio, los hijos de trabajadores del sector servicios, con un $32,7 \%{ }^{13}$.

Sería extraño, sin embargo, que la profesión por sí misma pudiese tener importancia como factor explicativo. Pienso, más bien, que la profesión es un índice de otros factores, tales como la complejidad y el grado de jerarquización de las estructuras de producción. Cuanto más complejas y jerarquizadas sean éstas, más necesarios resultan el contacto con la ciudad y la interacción con personas a las que se considera superiores en la escala social y que son, en la mayoría de los casos, castella-

${ }^{13} \underline{\mathrm{Ji}}^{2}$ para estos datos es 16,37 , significativa al nivel 0,01 . 
no-hablantes habituales. Como consecuencia de ello, las redes sociales son poco tupidas, con bajo grado de densidad y de multiplexidad ${ }^{14}$. En el extremo opuesto, cuanto menos complejas sean estas estructuras, las unidades de producción tienden a coincidir con unidades familiares, lo que facilita su repliegue sobre sí mismas y hace que sean más resistentes a los cambios de cualquier índole; las redes sociales en este tipo de estructuras productivas tienen un alto grado de densidad y de multiplexidad: todos los miembros de la red se interrelacionan, haciéndola asi cerrada, y las mismas personas mantienen múltiples contactos para actividades diversas (trabajan juntos, se divierten juntos, etc.). Milroy (1980) ha puesto de relieve la importancia que tiene este tipo de redes en el mantenimiento de las formas de hablar vernáculas, independientemente de lo estigmatizadas que estén, ya que el estigma queda compensado por el prestigio encubierto que poseen como elementos de identificacion de grupo y por el control social: hablar de una manera diferente de la del resto de los miembros de una red cerrada lleva necesariamente a la exclusión y a la integración en una red distinta. Lo mismo sucede en los procesos de sustitución lingüística. Si el gallego se ha mantenido con más firmeza en el campo que en la ciudad no es por ningún mérito heroico de los campesinos, sino porque el tipo de redes sociales que predominan hicieron necesaria su conservación. Del mismo modo, si lo rural se transforma y deja de ser exclusivamente agrícola, propiciando una mayor diversidad en los contactos y rebajando el grado de densidad y multiplexidad de las redes, el gallego deja de ser una necesidad absoluta, para pasar a convertirse en una necesidad relativa: sigue siendo necesario para cierto tipo de relaciones con miembros de la red, pero que ya no son los únicos con los que se establece relación.

La distancia a la ciudad no es ajena a estos procesos, en el sentido de que cuanto mayor sea la distancia, más probables serán las estructuras productivas poco complejas y escasamente jerarquizadas, así como las redes sociales inherentes a ellas; a medida que la distancia se vaya acortando, irán apareciendo estructuras más complejas y redes más abiertas, pero sin que desaparezcan del todo las más sencillas.

Este enfoque permite dar una explicación integradora de la diversidad de los datos rurales. Y si el enfoque es correcto, habrá que dejar de hacer afirmaciones acerca de la situación lingüística de las zonas rurales gallegas, en general; por el contrario, se necesitan muchas y muy detalladas

${ }^{14}$ El concepto de red social, su clasificación y la correlación de ciertos tipos de red con clases sociales y estructuras productivas son nociones que sólo en la última década se han incorporado a la sociolingüística, especialmente a partir de los trabajos de Milroy. Para una discusión de estos conceptos véase Boissevain (1974). 
descripciones de las diversas zonas, clasificadas según alguna medida de los factores que hemos considerado relevantes y atendiendo al grado $\mathrm{y}$ forma de inserción de sus habitantes en los mismos.

\section{Lengua materna y Clase Social}

La LM y la clase social están estrechamente relacionadas, como se puede observar en la figura 4. Prácticamente la totalidad de los jóvenes de clase social alta tienen como LM el castellano, excepto dos que aprendieron a hablar en las dos lenguas. En la clase media predomina el castellano, LM del 69,1\% de los jóvenes de ese grupo; el gallego lo es sólo del $15,6 \%$ y un porcentaje semejante (el 15,3\%) aprendió simultáneamente en las dos. En la clase social baja gallego y castellano presentan porcentajes casi iguales: $38,1 \%$ y $38,6 \%$ respectivamente, y un $23 \%$ aprendió a hablar en las dos ${ }^{15}$.

Una vez más nuestros datos ofrecen porcentajes claramente más desfavorables al gallego que las encuestas anteriores. Ayestarán y de la Cueva (1974), por ejemplo, ordenan a las amas de casa de su encuesta según sus ingresos económicos mensuales. Los porcentajes de encuesta-

\section{LENGGU MATERNA SEGÚN CLASE SOCIAL}

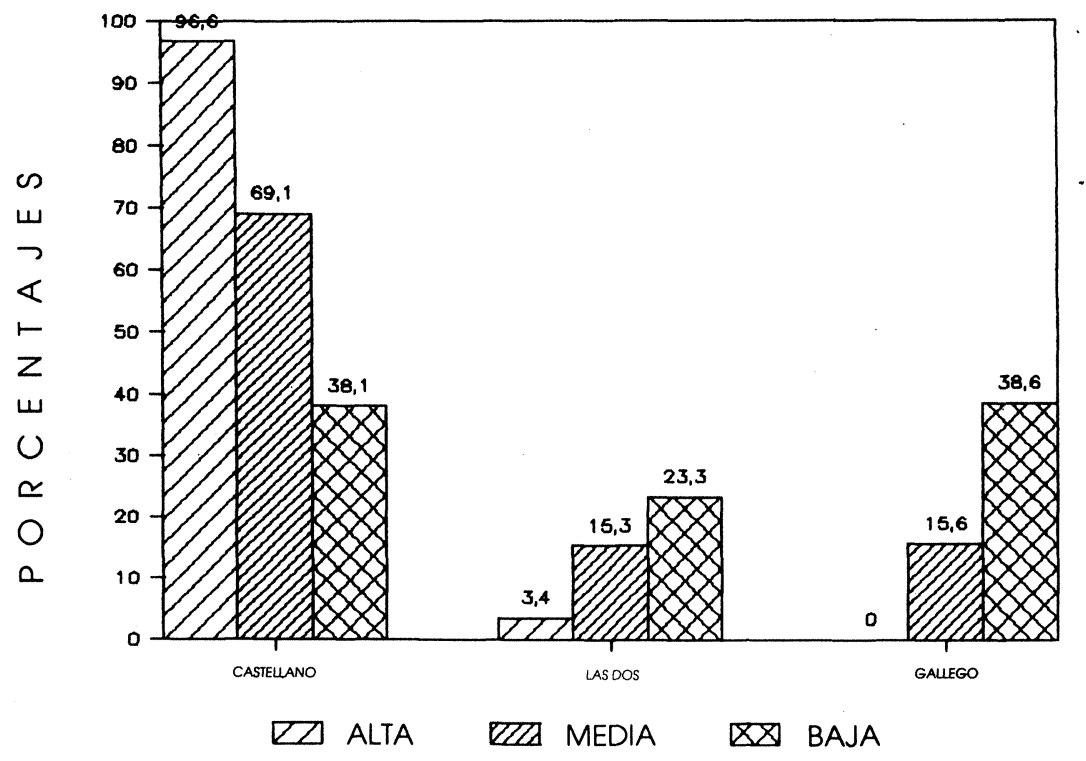

${ }^{15} \underline{\mathrm{Ji}}^{2}=123,07$ (significación $\left.=0,0000\right)$ y $\underline{\mathrm{V}}$ de Cramer $=0,27$. 
das con el gallego como LM van decreciendo, desde el $95 \%$ en el grupo económicamente inferior hasta el $47 \%$ en la clase media alta ${ }^{16}$.

Es necesario destacar que el porcentaje más bajo obtenido por esos autores es tres veces superior al obtenido por mí en los jóvenes de clase social media, y es superior, incluso, al obtenido en la clase social baja. No cabe duda, pues, de que la desalleguización en cuanto a la LM abarca a todas las clases sociales.

\section{Lengua materna según Clase Social y Hábitat}

Sobradamente conocida es la correlación existente en Galicia entre clase social y tipo de habitat ${ }^{17}$. Es necesario, por consiguiente, matizar un poco más lo dicho en los puntos 3 y 4 .

En las figuras - A y $5 \mathrm{~B}$ se presentan los resultados de cruzar la $\mathrm{LM}$ con la clase social según el tipo de hábitat. Los datos relativos a la clase social

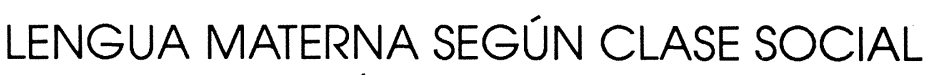
HÁBITAT URBANO

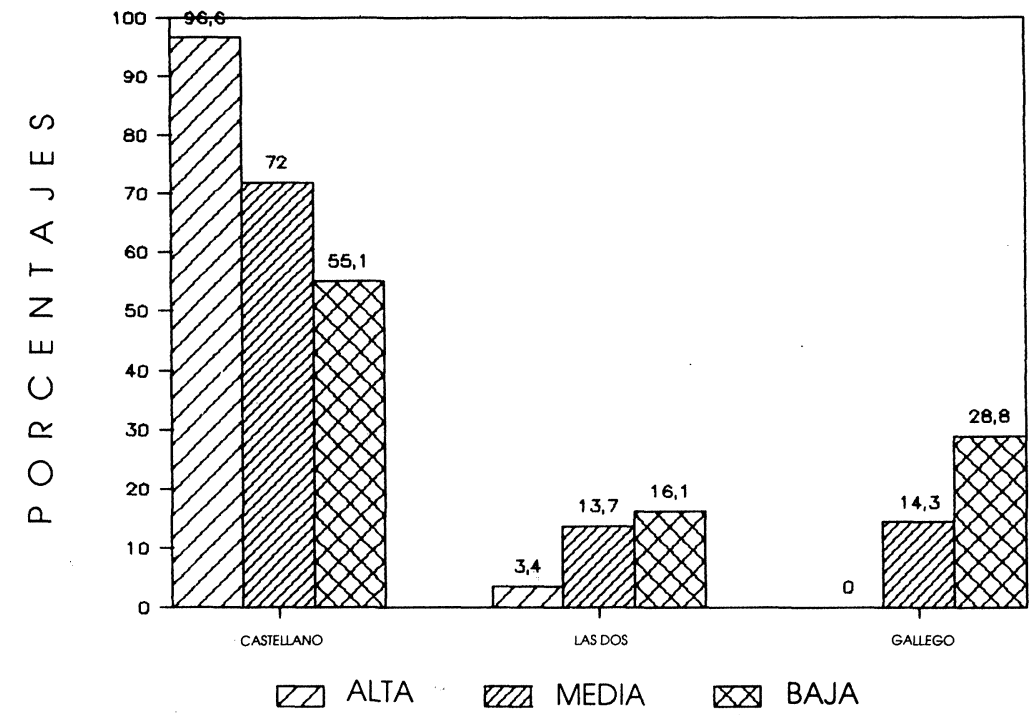

${ }^{16} \mathrm{El}$ grupo que obtenía en el año de la encuesta de 30.000 a 50.000 pts. mensuales (datos deducidos del cuadro 33, p. 88). Aunque el grupo con ingresos superiores a 50.000 pesetas mensuales presenta un porcentaje de GHI del $67 \%$, no es posible tomarlo en consideración, ya que ese porcentaje se ha calculado sobre tres casos.

17 Todos los jóvenes de clase alta en la muestra son urbanos, así como el 89,9\%de los de clase media; la clase baja está más repartida: el 49,8\% urbanos y el 50,2\% rurales. Desde la perspectiva complementaria, el $84,8 \%$ de los niños rurales son de clase social baja y el $15,2 \%$ de clase media, frente al $34,6 \%$ y $55,5 \%$, respectivamente, en la ciudad.

"CUADERNOS DE ESTUDIOS GALLEGOS", Tomo XXXIX, Fascículo 104, Santiago 1991. 


\section{LENGUA MATERNA SEGÚN CLASE SOCIAL}

HÁBITAT RURAL

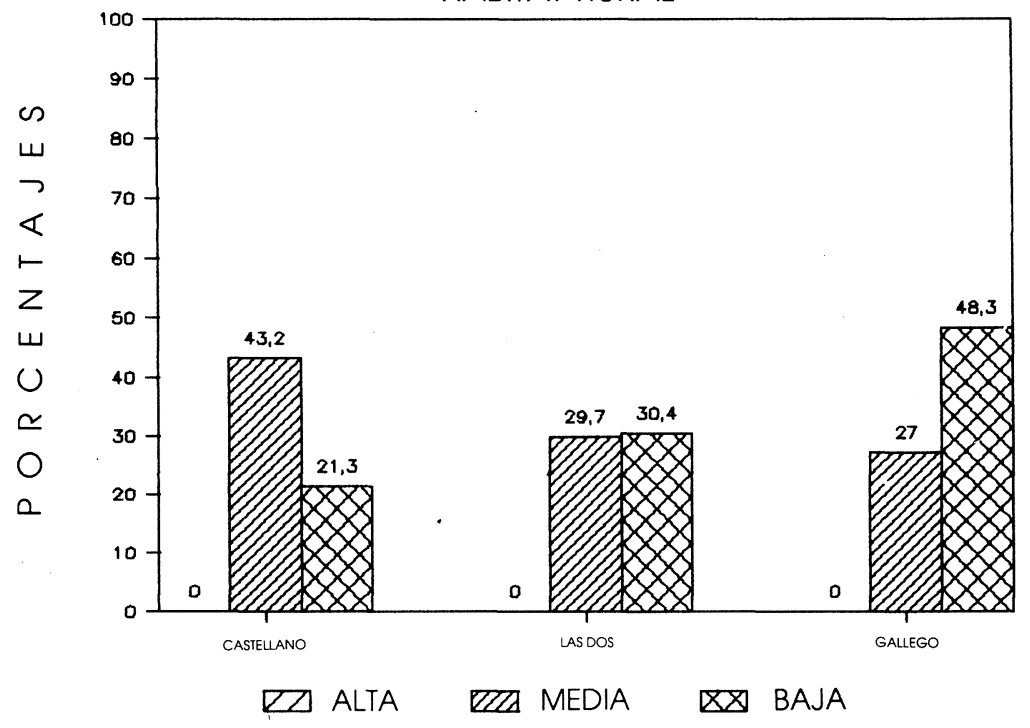

alta no sufren, lógicamente, ninguna modificación, ya que todos los jóvenes de esta clase social incluidos en la muestra pertenecen a un hábitat urbano; asimismo, no serán grandes las diferencias entre los datos ya presentados para el conjunto de la clase media y los de la clase media urbana, puesto que los jóvenes rurales son solamente el 10,15\% de esta clase social; por consiguiente, por mucho que estos últimos se distancien del promedio de su clase, la repercusión en los datos urbanos será pequeña. En efecto, un $72 \%$ de los jóvenes urbanos de clase social media aprendieron a hablar primero en castellano, porcentaje muy ligeramente superior al promedio de esta clase prescindiendo del tipo de hábitat $(69,1 \%)$. Las otras dos opciones están ligeramente por debajo del promedio: un 13,7\% aprendieron a hablar en las dos lenguas (el promedio es de $15,3 \%$ ) y un $14,3 \%$ son GHI (el porcentaje promedio es 15,6\%) Inversamente, estas pequeñas diferencias con el promedio en la clase media urbana se traducirán en diferencias notables en los jóvenes rurales de la misma clase: aprendieron a hablar primero en castellano el $43,2 \%$, veintiséis puntos por debajo del porcentaje promedio; las otras dos opciones se sitúan alrededor de quince puntos por encima del promedio: son GHI el $27 \%$ de los jóvenes, y el 29,7\% aprendió a hablar simultáneamente en las dos lenguas. No obstante lo espectacular de estas diferencias, el escaso tamaño de la submuestra rural hace que no sean estadísticamente significativas. 
Los jóvenes de la clase social baja se reparten casi al $50 \%$ entre los dos tipos de hábitat, pero como el $84,4 \%$ de los jóvenes rurales pertenecen a esta clase social, los resultados que se obtengan al cruzar la LM con las dos variables no serán muy diferentes de los obtenidos para el conjunto de los jóvenes rurales, aunque podrán diferir bastante de los obtenidos para el conjunto de la clase social baja sin controlar el tipo de hábitat. Efectivamente, un $48.3 \%$ aprendieron a hablar primero en gallego, porcentaje que es superior en diez puntos al promedio para esta clase $(38,1 \%)$ y sólo tres puntos superior al promedio rural $(45,1 \%)$. El castellano es la LM del 21,3\% de los jóvenes, cuatro puntos por debajo del promedio de la clse social. El 30,4\% de los jóvenes aprendieron a hablar simultáneamente en las dos lenguas, porcentaje semejante al promedio rural, pero siete puntos por encima del promedio de la clase social. Lo contrario sucede en la ciudad, en donde los datos obtenidos para la clase social baja están a considerable distancia tanto del promedio de su clase como del de su tipo de hábitat. Son $\mathrm{CHI}$ el $55,1 \%$ de los jóvenes, porcentaje superior en diecisiete puntos al promedio de su clase $(38,1 \%)$ e inferior en casi catorce puntos al de su hábitat $(68,8 \%)$; son GHI el $28,8 \%$, porcentaje que es algo más de diez puntos inferior al promedio de su clase social $(38,1 \%)$ y once puntos superior al de su hábitat $(17,9 \%)$; aprendieron a hablar en las dos lenguas el $16,1 \%$, siete puntos por debajo del promedio de la clase $(23,3 \%)$ y tres puntos por encima del promedio de su hábitat $(13,5 \%)$.

En contraste con lo observado enla clase media, las diferencias entre la clase social baja rural y la urbana son significativas ${ }^{18}$. También lo son asimismo las diferencias entre las clases sociales en ambos tipos de hábitat ${ }^{19}$.

Resulta difícil determinar con cuál de las dos variables que estamos considerando se asocia más la LM. Si las diferencias entre las clases sociales son significativas en los dos tipos de hábitat, mientras que las diferencias de hábitat sólo son significativas en la clase social baja, parecería ser mayor la asociación entre LM y clase social que la existente entre LM y hábitat. Sin embargo, tal vez nos encontramos ante una pura apariencia. Si consideramos las tres variables como escalas ordinales ${ }^{20}$, es posible

${ }^{18} \underline{\mathrm{Ji}}^{2}=50,27$ (significación $\left.=0,0000\right)$ y $\underline{\mathrm{V}}$ de Cramer $=0,35$.

${ }^{19}$ En el hábitat rural: $\underline{\mathrm{Ji}}^{2}=9,83$ (significación $=0,0094$ ) y $\underline{\mathrm{V}}$ de Cramer $=0,20$. En el hábitat urbano $\underline{\mathrm{Ji}}^{2}=44,80$ (significación $=0,0000$ ) y $\underline{\mathrm{V}}$ de Cramer $=0,19$.

${ }^{20}$ Las categorías utilizadas implican, en rigor, un nivel de medición nominal. No obstante, si nos atenemos a la mayor o menor presencia inicial de una de las lenguas, podemos asumir un nivel de medición ordinal en el que gallego $>$ las dos $>$ castellano (o a la inversa, si se atiende a la mayor o menor presencia inicial del castellano). Aplicando el mismo razonamiento a la clase social y al hábitat, se pueden utilizar coeficientes de correlación de rangos.

"CUADERNOS DE ESTUDIOS GALLEGOS", Tomo XXXIX, Fascículo 104, Santiago 1991. 
utilizar el coeficiente gamma en diversos órdenes. Gamma de orden cero indica la correlación existente entre dos variables sinconsiderar la influencia de una tercera; gamma de primer orden elimina de la correlación la parte debida a la influencia de la tercera variable. Es decir, de lo que se trata es de suprimir de la correlación entre lengua y hábitat lo que éste tiene de indicador de clase social; del mismo modo, habrá que suprimir de la correlación entre lengua y clase social la asociación que ésta tiene con el hábitat. Los coeficientes son los siguientes:

\section{LM y clase social}

gamma de orden cero

0,59561

gamma de primer orden (controlando hábitat)

0,46480

Diferencia

\section{LM y hábitat}

gamma de orden cero

gamma de primer orden (controlando clase social)

0,62988

Diferencia

Como se puede observar, las dos gammas correspondientes al cruce de la LM con el hábitat son mayores que las del cruce con la clase social; pero la que interesa especialmente es gamma de primer orden, ya que supone que se ha eliminado la correlación con la variable que se controla, cuya influencia viene expresada por la diferencia entre las gammas de los dos órdenes; esta diferencia es mayor en el cruce con la clase social. Por consiguiente, el hábitat explica una parte de la relación entre LM y clase social mayor que la explicada por la clase social en la relación de la LM con el hábitat. La asociación de la LM con el hábitat es,pues, mayor que con la clase social. La apariencia en contra se debe a que las diferencias entre los tipos de hábitat sólo son significativas en una clase social: la baja; pero se trata de las diferencias más significativas de todas las analizadas, con un coeficiente $V$ de Cramer de 0,35 .

\section{Lengua materna y edad}

La edad es otra de las variables que la sociolinguística suele tomar en cuenta en el estudio del cambio de lengua en una población. En ella se basan, naturalmente, las comparaciones entre generaciones efectuadas en apartados anteriores, que muestran la existencia de un proceso de desgalleguización considerable, en lo que a LM se refiere. Pero no esperábamos que la desgalleguización se pudiese apreciar con intervalos de edad tan pequeños como los que nosotros utilizamos. Las edades de los jóve- 
nes de nuestra muestra iban, en el momento de la recogida de datos, desde los diez hasta los catorce años. Prescindiendo de los jóvenes de diez años (solamente seis tenían esa edad), observamos en la figura 6 cómo la desgalleguización se hace patente de año en año. Los porcentajes de castellano-hablantes van disminuyendo a medida que va aumentando la edad, con veinte puntos de diferencia entre los extremos: entre los de once años son CHI el 64,1\%, el 59\% entre los de doce, el 52,5\% entre los de trece y el $44 \%$ entre los de catorce. Correlativamente, los porcentajes de GHI van aumentando con la edad: el $22,5 \%$ entre los de once años, el 23,8\% entre los de doce, el $26,7 \%$ entre los de trece y el $32,7 \%$ entre los de catorce. La misma pauta se observa entre los $\mathrm{BI}^{21}$.

En la muestra hay un sesgo en la relación entre edad y clase social: el $63,5 \%$ de los que tenían catorce o más años son de clase social baja, debido a la mayor incidencia del "fracaso escolar" en este grupo social, que es el principal proveedor de repetidores. Es necesario, por consiguiente, controlar esta última variable. Aunque la significación estadística desapa-

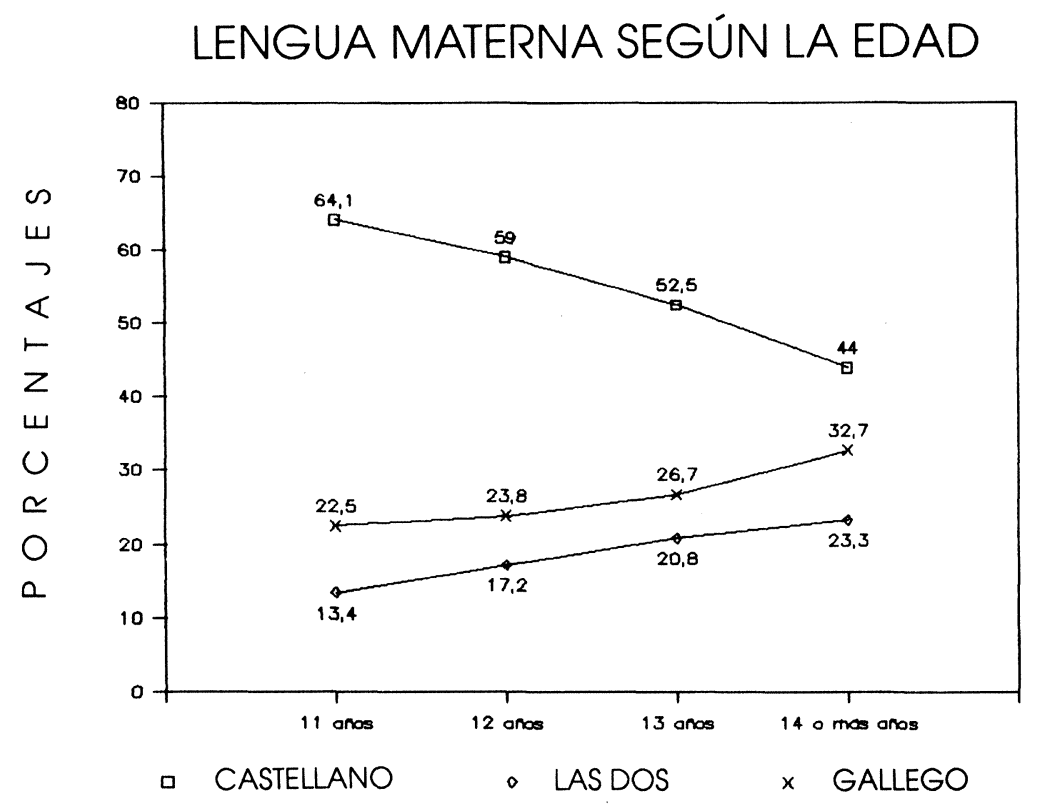

${ }^{21} \mathrm{uJi}^{2}=\mathrm{Ji}^{2}=17,44$ (significación $=0,0078$ ). De todos modos, la fuerza de la correlación es baja: $\underline{\mathrm{V}}$ de Cramer $=0,10$.

"CUADERNOS DE ESTUDIOS GALLEGOS", Tomo XXXIX, Fascículo 104, Santiago 1991. 
rece al introducir este control, me parece importante resaltar que la tendencia se sigue observando en la clase social baja, como se puede observar en la figura 7 . El porcentaje de CHI es el 48,4\% en los jóvenes de once años, el $42,2 \%$ en los de doce, el $32,4 \%$ en los de trece y el $28,7 \%$ entre los de catorce. El porcentaje de GHI es el 32,6\% entre los de once años, el 38,2\% en los de doce, el 39,6\% en los de trece y el 44,6\% en los de catorce 22 . La pérdida del gallego en cuanto LM es, pues, tan considerable en la clase social baja que sus efectos se pueden observar con intervalos de un año.

\section{Conclusiones}

Los datos presentados en este trabajo muestran un importante descenso del gallego como LM en comparación con los datos de que disponemos para la población adulta, descenso que afecta también a los sectores de la población que tradicionalmente habían venido manteniendo esta lengua.

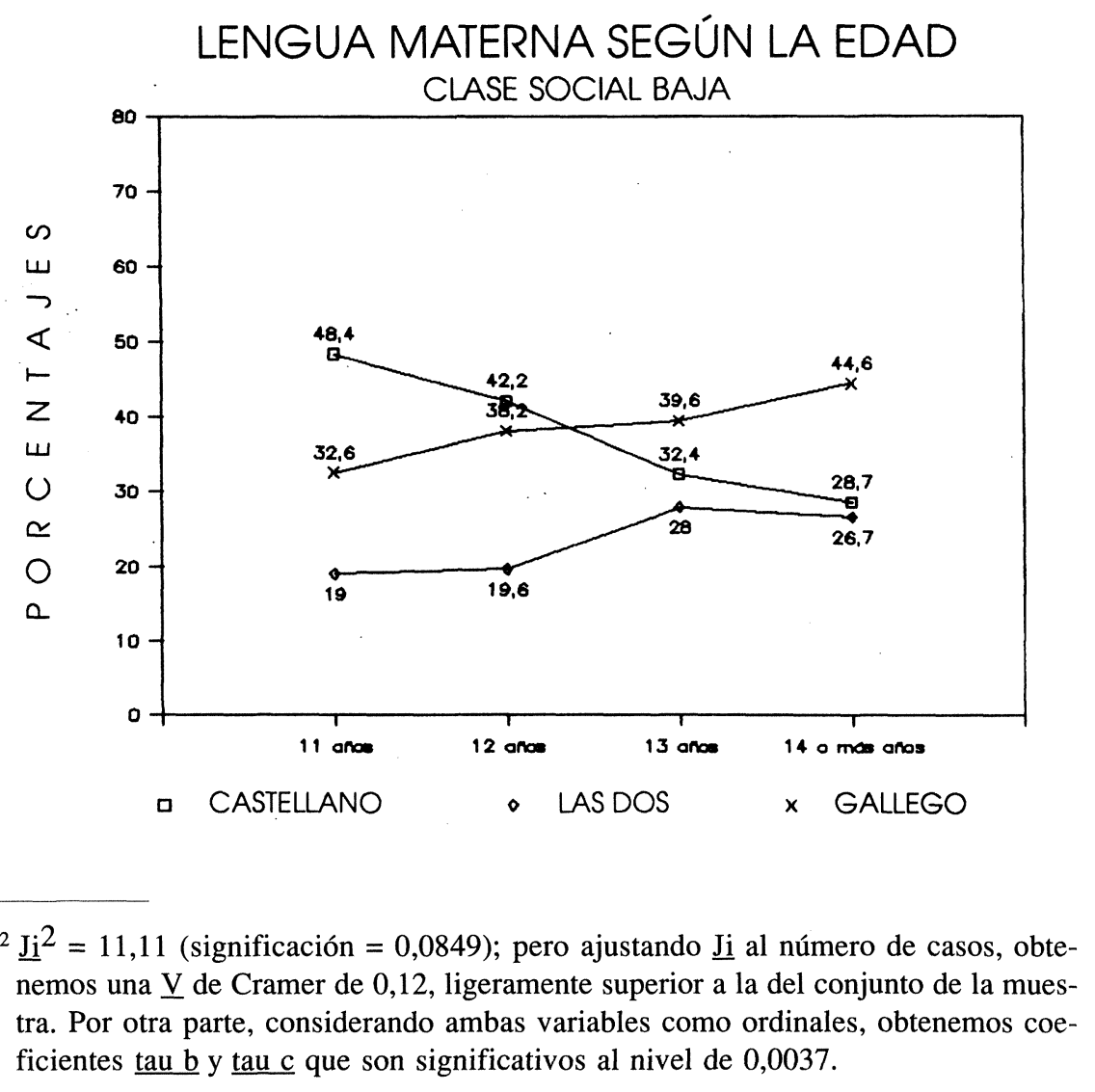

"CUADERNOS DE ESTUdIOS GALLEGOS", Tomo XXXIX, Fascículo 104, Santiago 1991. 
De entre todos los datos presentados, hay dos que quisiéramos destacar. En primer lugar, el porcentaje más eleado de GHI en la muestra corresponde a los jóvenes de clase social baja que son hijos de agricultores; pero este porcentaje es solamente algo más de la mitad: el 53,2\%, veinticuatro puntos inferior al obtenido por Ayestarán y de la Cueva entre las amas de casa rurales del municipio de Vigo. En segundo lugar, el proceso de desgalleguización en cuanto a la LM es tan intenso y tan constante que sus resultados se hacen notar no sólo entre una generación y la que le sigue, sino dentro de una misma generación, con intervalos de edad de solamente un año.

El proceso de desgalleguización adopta a veces una forma intermedia, que consiste en enseñar a hablar al niño en las dos lenguas simultáneamente. Esta forma intermedia puede considerarse bien como el fracaso de una estrategia dirigida a la desgalleguización total, bien como una resistencia frente a la misma. Lo que parece evidente es que, salvo casos esporádicos, el bilingüismo inicial no es el producto de un proceso de regalleguización, ya que los porcentajes más elevados de BI se dan en los sectores tradicionalmente menos castellanizados. Sea fracaso en la desgalleguización o resistencia a la misma, es importante tener en cuenta que el porcentaje de BI disminuye con la edad, lo mismo que el de GHI.

En conjunto, hay una asociación más intensa de la LM con el hábitat que con la clase social. Pero es necesario tener en cuenta que el hábitat rural es menos homogéneo de lo que habitualmente se supone: de solamente un $6 \%$ de CHI entre los hijos de agricultores pasamos a porcentajes entorno al $30 \%$ en los hijos de obreros y en los de trabajadores del sector servicios, porcentajes próximos al correspondiente a los jóvenes urbanos de clase social baja.

Partiendo de la LM, el proceso de castellanización (o desgalleguización) es, pues, muy intenso. De todos modos, como ya he señalado, es muy cuestionable que en Galicia sea la LM el mejor índice de desgalleguización. Los datos sobre conocimiento del gallego y sobre su utilización (si huimos de dicotomías gallego/castellano) permiten obtener un panorama un poco más favorable para el gallego; pero no mucho más. 


\section{REFERENCIAS BIBLIOGRÁFICAS}

AYESTARÁN, M. y J. DE LA CUEVA, 1974., Las familias de la provincia de Pontevedra en 1974. Sevilla, Instituto de Ciencias de la Familia.

BOISSEVAIN, J. 1974. Friends of Friends: Networks, Manipulators and Conditions. Oxford, Blackwell.

DEL CAMPO, S., M. NAVARRO y J.F. TEZANOS 1977. La cuestión regional española. Madrid, Edicusa.

DIRECCIÓN XERAL DE POLÍTICA LINGÜÍSTICA (1986). Aspectos sociolingüísticos do bilingüismo en Galicia segundo os alumnos da $2^{\underline{a}}$ etapa de E.x.B. Xunta de Galicia.

FERNÁNDEZ, M. 1978. bilingüismo y Planificación Lingüística en Galicia. Tesina (inédita), Universidad de Santiago.

FERNÁNDEZ, M. 1983. "Mantenimiento y cambio de lengua en Galicia: el ritmo de la desgalleguización en los últimos cincuenta años". Verba 10. 79-129.

FERNÁNDEZ, M. 1984. Conocimiento, uso y actitudes lingüísticos de los alumnos de EGB del municipio de Santiago. Tesis Doctoral (inédita), Universidad de Santiago.

FOESSA 1970. Informe sociológico sobre la situación actual de España: 1970. Madrid, Fundación Foessa-Euramérica.

LIEBERSON, S. (1980). "Procedures for improving sociolinguistic surveys of language maintenance and language shift". International Journal of the Sociology of Language, 25, 11-27.

MILROY, L. 1980. Language and Social Networks. Oxford, Blackwell.

SKUTNABB-KANGAS, T. 1984. Bilingualism or not: the education of minorities. Clevedon, Multilingual Matters.

SKUTNABB-KANGAS, T. 1988. "Multilingualism and the education of minority children". En T. Skutnabb-Kangas y J. Cummins (eds.), Minority Education. Clevedon, Multilingual Matters.

"CUADERNOS DE ESTUDIOS GALLEGOS", Tomo XXXIX, Fascículo 104, Santiago 1991. 